\title{
Static Traction and Early Mobilization Protocol for Intra-articular Fractures of Proximal Interphalangeal Joint: Technique and Outcome
}

\author{
Nilesh D Darawade ${ }^{1}$, Praveen Bhardwaj ${ }^{2}$, Raja Sabapathy Shanmuganathan ${ }^{3}$
}

\begin{abstract}
Aim and background: Intra-articular fractures of the proximal interphalangeal (PIP) joint are a difficult entity to treat, commonly leading to stiff and painful fingers. There are multiple techniques described for treatment. We utilize a simple technique consisting of a static traction assembly and early mobilization of the finger to treat this pathology. In this study, we present results obtained with this modality.

Materials and methods: We utilize a malleable aluminum splint for traction against the injured finger. No. 1 polypropylene suture through nail plate of injured finger applies traction to hold joint in reduced position for 2 weeks. At 2 weeks, active-assisted mobilization of the injured finger is begun. Patients were evaluated for the range of motion achieved, radiograph picture, presence of pain, and ability to return to work. Results: There were 11 cases of intra-articular fractures of the middle phalanx base. The median period of follow-up was 15 months (range $7.5-31$ months). The average active flexion possible at the injured joint was $87.7^{\circ}\left(\right.$ range $\left.55-110^{\circ}\right)$. Two patients reported mild pain on terminal flexion without functional limitations. In unstable injuries, suboptimal alignment of fracture was common [9 out of 11]. Despite suboptimal radiographs, patients had a functional, painless range of motion. All patients went back to previous work.

Conclusion: Static traction technique with early mobilization protocol is a safe, relatively simple, and effective technique in the management of intra-articular fractures of the PIP joint. Early mobilization of joint facilitates remodeling of the articular base of the middle phalanx with the recovery of painless motion at the PIP joint despite radiological abnormalities.

Keywords: Fracture-dislocation, Mobilization protocol, Proximal interphalangeal joint, Static traction.

Journal of Orthopedics and Joint Surgery (2022): 10.5005/jp-journals-10079-1050
\end{abstract}

\section{INTRODUCTION}

Intra-articular fracture-dislocation of the proximal interphalangeal (PIP) joint has been a challenging problem for hand surgeons. It has been described as "one of the unanswered and perhaps unanswerable questions of hand surgery." ${ }^{11}$ We present results of a nail plate static traction technique with early mobilization protocol followed at our center. The technique has been described as a method of conservative management of fractures in fingers. ${ }^{2}$ There have been many techniques described in the literature to treat this difficult condition such as extension block splint ${ }^{3} /$ pinning ${ }_{1}^{4}$ dynamic traction systems with outriggers ${ }^{5,6}$ or pins and rubber, ${ }^{7-12}$ volar plate arthroplasty, ${ }^{13}$ internal fixation of fracture, ${ }^{14,15}$ and hemi-hamate arthroplasty. ${ }^{16}$ Many of them are technically demanding. We present results of 11 patients treated with static traction technique and early mobilization protocol with an average follow-up of 17.3 months.

\section{Materials and Methods}

Twenty consecutive patients with intra-articular fractures of the PIP joint were treated with static traction and early mobilization protocol from April 2013 to July 2015. Nineteen of these patients had a fracture of the volar base of the middle phalanx with dorsal dislocation of the PIP joint and one patient had a pilon fracture of the base of the middle phalanx. All the patients with intra-articular fractures of the PIP joint, irrespective of the degree of comminution, presenting up to 2 weeks from injury were offered this modality of treatment.
${ }^{1}$ Department of Hand and Microsurgery, Deenanath Mangeshkar Hospital, Pune, Maharashtra, India

${ }^{2,3}$ Department of Plastic, Hand and Reconstructive Microsurgery, Ganga Medical Centre and Hospitals, Coimbatore, Tamil Nadu, India

Corresponding Author: Nilesh D Darawade, Department of Hand and Microsurgery, Deenanath Mangeshkar Hospital, Pune, Maharashtra, India, Phone: +91 9894006697, e-mail: drnilesh1@gmail.com

How to cite this article: Darawade ND, Bhardwaj P, Shanmuganathan RS. Static Traction and Early Mobilization Protocol for Intra-articular Fractures of Proximal Interphalangeal Joint: Technique and Outcome. J Orth Joint Surg 2022;4(1):8-13.

Source of support: Nil

Conflict of interest: None

\section{Operative Technique}

The procedure was done under the brachial plexus block. Under fluoroscopic control, the fracture dislocation was reduced by longitudinal traction and flexion of the joint. The aim was to get the fracture fragments into the best possible alignment by molding, using the soft tissue envelope around the site of the fracture and correct joint subluxation. We did not attempt any percutaneous/ invasive technique for fracture reduction. A preliminary reduction also indicated the amount of traction needed to maintain joint alignment and the degree of flexion required.

The material needed for the traction assembly is-plaster roll, malleable aluminum splint, no. 1 polypropylene suture, cotton bandages, and adhesive tape (Fig. 1A ). The splint is bent by $20-30^{\circ}$

() The Author(s). 2022 Open Access This article is distributed under the terms of the Creative Commons Attribution 4.0 International License (https://creativecommons. org/licenses/by-nc/4.0/), which permits unrestricted use, distribution, and non-commercial reproduction in any medium, provided you give appropriate credit to the original author(s) and the source, provide a link to the Creative Commons license, and indicate if changes were made. The Creative Commons Public Domain Dedication waiver (http://creativecommons.org/publicdomain/zero/1.0/) applies to the data made available in this article, unless otherwise stated. 
to keep wrist extended, another bend of $90^{\circ}$ is made at the level of distal palmar crease along the long axis of injured finger to keep metacarpophalangeal joint in flexion. After molding the splint, it is incorporated between two layers of plaster roll (Fig. 1B ). It is necessary to wait till plaster sets before proceeding further. At this stage, a no. 1 polypropylene suture is passed through the nail plate to hold $0.8-1 \mathrm{~cm}$ of the nail plate for secure fixation (Fig. 1C). The needle should be passed parallel to the nail plate to avoid injury to the nail bed. If the needle is passed accordingly, bleeding should be absent or minimal. The malleable splint is then bent dorsally, about $2-4 \mathrm{~cm}$ beyond the fingertip to create a post for traction (Fig. 1D). Traction is applied again to reduce the PIP joint by holding the fingertip. It is important not to apply traction by pulling the thread to avoid thread cutting through the nail plate. The purpose of the thread is to maintain traction achieved by the above-mentioned technique. Once traction is sufficient, the thread is tied around the post created by a malleable splint and held with adhesive tapes applied on knots. Reduction may be confirmed again under fluoroscopy but we have not found it necessary and do it only occasionally.

\section{Postoperative Care}

Patients were instructed regarding the care of splint and discharged the same day. A follow up was done at 1 week postoperative period to assess the status of the splint. Traction was discontinued at 12-14 days by dividing and removing thread in the outpatient department. Usually, by this time, there was no tenderness at the fracture site. The splint was bent away from the finger to allow for active-assisted finger mobilization which was started at this stage. The below-elbow plaster splint was retained for 1 more week for ease of mobilization and to prevent active use of the hand. At 3 weeks postoperatively, plaster splint was discontinued and patients were instructed to continue active-assisted mobilization of PIP and distal interphalangeal (DIP) joints. At 6 weeks postoperatively, patients could start active use of the hand for light activity, unrestricted activity resumed at 10 weeks postoperatively. Patients were reviewed at 2 weeks, 3 weeks, 6 weeks, 3 months, 6 months, 1 year, and 2 years postoperatively.

\section{Follow-up Evaluation}

The range of motion of the PIP and DIP joints was measured with a goniometer, true lateral radiographs evaluated and patients
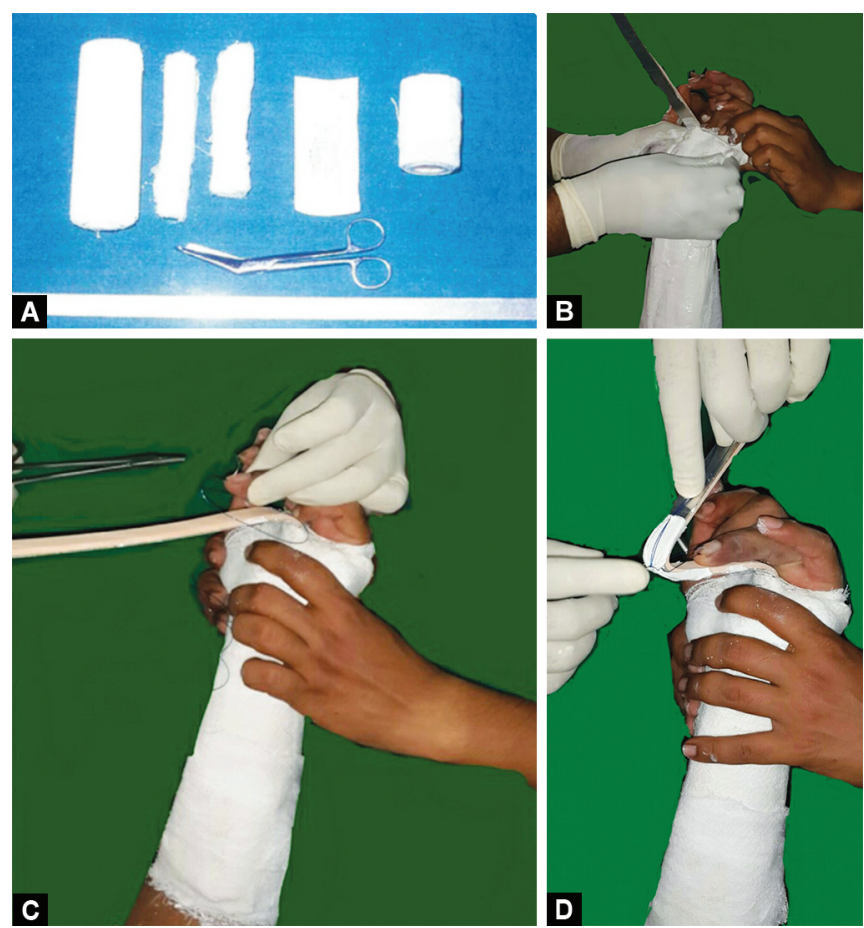

Figs 1 A to D: (A) Materials required for construction of the assembly; (B) Malleable splint incorporated within two layers of plaster; (C) Number 1 prolene being passed through nail plate for traction; (D) Completion of construct by tying thread to post created in a splint. Note traction is not applied via thread but by pulling on the fingertip to reduce joint, thread maintains traction thus created

were interviewed for subjective assessment of pain, return to the previous level of activity.

\section{Results}

We had a total of 20 patients in this series. Eight patients were lost in follow-up. They had not reported for follow-up visits beyond 6 weeks but their physiotherapy records revealed a progressively increasing range of painless flexion before they discontinued their visit to the hospital. One patient regarded the result as poor

Table 1: Patient data and outcome measures

\begin{tabular}{|c|c|c|c|c|c|c|c|}
\hline Case no. & Age (years) & Injured finger & Type of injury & $\begin{array}{c}\% \text { Articular } \\
\text { surface involved }\end{array}$ & $\begin{array}{c}\text { Delay } \\
\text { between injury } \\
\text { and surgery } \\
\text { (days) }\end{array}$ & $\begin{array}{c}\text { Active range of } \\
\text { motion of PIP } \\
\text { joint at the final } \\
\text { review }\end{array}$ & $\begin{array}{l}\text { FFD/extension lag at } \\
\text { either IP joints }\end{array}$ \\
\hline 1 & 27 & Left middle & Volar base fracture & 45 & 1 & $15-95^{\circ}$ & $\mathrm{PIP}-10^{\circ} \mathrm{FFD}$ \\
\hline 2 & 36 & Left index & Volar base fracture & 73 & 1 & $0-105^{\circ}$ & DIP-10 Ext. lag \\
\hline 3 & 26 & Left ring & Volar base fracture & 26 & 0 & $0-95^{\circ}$ & - \\
\hline 4 & 28 & Left ring & Volar base fracture & 33 & 1 & $0-95^{\circ}$ & - \\
\hline 5 & 62 & Left little & Volar base fracture & 50 & 4 & $5-75^{\circ}$ & $\begin{array}{c}\text { PIP- } 5^{\circ} \text { ext. lag, DIP- } \\
15^{\circ} \text { ext. lag }\end{array}$ \\
\hline 6 & 40 & Left ring & Volar base fracture & 57 & 1 & $10-100^{\circ}$ & PIP $-10^{\circ}$ ext. lag \\
\hline 7 & 23 & Left index & Volar base fracture & 46 & 14 & $10-55^{\circ}$ & $\mathrm{PIP}-10^{\circ}$ ext. lag \\
\hline 8 & 26 & Right ring & Volar base fracture & 45 & 3 & $0-90^{\circ}$ & DIP-15e ext. lag \\
\hline 9 & 61 & Left ring & Pilon fracture & 100 & 0 & $20-85^{\circ}$ & $\mathrm{PIP}-20^{\circ} \mathrm{FFD}$ \\
\hline 10 & 21 & Left middle & Volar base fracture & 46 & 10 & $0-85^{\circ}$ & $\mathrm{DIP}-25^{\circ}$ ext. lag \\
\hline 11 & 20 & Left ring & Volar base fracture & 39 & 0 & $0-100^{\circ}$ & DIP-10 $10^{\circ}$ ext. lag \\
\hline
\end{tabular}


with a stiff finger on telephonic conversation and was unavailable for clinical assessment. Rest 11 patients with a median follow-up period of 15 months (range 7.5-31 months) form part of this study. Table 1 shows the demographic details of these patients. All affected patients were males. The average age of patients was 33.6 years (range 20-62 years). One patient suffered an injury in the dominant hand, rest 10 in the non-dominant hand. Six patients had ring finger injuries, the index finger was involved in two patients, the long finger in two, and the little finger in one patient (Table 1). The average duration between injury and treatment was 3.08 days (range $0-14$ days).

The average articular surface involved in fracture of base of the middle phalanx was $51 \%$ (range $26-100 \%$ ) [Table 1].

The average active arc of motion achieved was $83.6^{\circ}$ (range $45-105^{\circ}$ ). The average loss of active extension at the PIP joint was $5.4^{\circ}$; three patients had extension lag of $5-10^{\circ}$; and two patients had flexion deformities of $10^{\circ}$ and $20^{\circ}$. Flexion at the PIP joint averaged $87.7^{\circ}$; with a minimum of $55^{\circ}$ and a maximum of $105^{\circ}$. Five patients had extension lag at DIP joints averaging $15^{\circ}$ (Table 1). Two patients reported mild pain on terminal flexion of the injured joints but could carry out activities of daily living without any medications. Three patients had lateral deviation of fingers at injury site averaging $18^{\circ}$ without rotation deformity. On lateral radiographs, there was a residual dorsal translation of the axis of the middle phalanx compared with the axis of the proximal phalanx in six patients (Fig. 2). All patients went back to their preinjury activities.

\section{Discussion}

Fracture dislocations of PIP joints are prone to cause a stiff painful finger if concentric movements of joints are not restored. One stiff finger can impair the function of the entire hand and jeopardize a patient's career. ${ }^{17}$ The goals of treatment are-to obtain a concentric reduction of PIP joint, to maintain joint stability, to re-establish gliding motion, and to allow early motion. ${ }^{18}$ Treatment by traction devices is based on two principles-ligamentotaxis through traction restores joint and fracture alignment; early mobilization promotes joint healing and restores function. ${ }^{6}$ Techniques involving the traction method can be dynamic or static. Dynamic methods allow motion with the traction device in situ. ${ }^{5,6,8}$ The technique described by Schenck ${ }^{5}$ and its modifications involves placement of pin in a middle phalanx with the application of outrigger incorporated in a cast allowing free movement, although a dorsal extension block may be added to prevent complete extension of the joint. The PIP joint needs to be at the center of the circle formed by the outrigger. Recently, dynamic traction device using pins and rubber described by Slade et al. ${ }^{8}$ seems to have gained wide acceptance with many articles describing good results. ${ }^{9-12}$ This technique involves placing a pin through the center of rotation of the head of the proximal phalanx which is the most critical step followed by two pins through the middle phalanx and connecting the hooked wires with dental rubber bands. Both these methods need precise placement of pins and meticulous construction of the assembly. Insertion of $k$-wires is subject to the risk of infection although the need for removal of fixator due to infection is rare and management with oral antibiotics usually suffices. Static external fixation can be utilized as a step before operative reduction and fixation of PIP joint ${ }^{19}$ or as a definitive treatment. ${ }^{20}$ Using a static fixator obviates the need for a complex outrigger system. Stark ${ }^{20}$ cited a reduction in the immobilization period to 3 weeks as an advantage of the static external fixator. With traction assembly in our series, using nail plate for traction application spared the need of $k$ wires in phalanges with consequent possibilities of infection at pin site. We routinely remove traction at 2 weeks, even further reducing the period of immobilization.

A review of the literature allows the comparison of our results with those obtained with alternative surgical techniques (Table 2). The ring finger was the most commonly involved finger in our series which corroborates with literature..$^{10,21,25}$ Dynamic traction systems involving $K$-wires and rubber band construct demonstrated mean PIP joint flexion achieved in the range of $82-89^{\circ}$. 6,9-12 Badia et al. $^{22}$ reported the use of a dynamic fixator without rubber bands with an average range of motion achieved from $5^{\circ}$ to $89^{\circ}$. After closed reduction and trans-articular pinning, a mean ROM of $85^{\circ}$ was reported in a series of 11 unstable fracture-dislocations at a mean follow-up of 16 years. ${ }^{23}$ In a study of extension block pinning with a follow-up of an average of 5.2 years, $80^{\circ}$ mean range of motion has been reported for 39 injured fingers in 37 patients for unstable dorsal fracture-dislocations of PIP joint. ${ }^{21}$ After open reduction and internal fixation with mini-screw for the base of the middle phalanx, the average arc of motion achieved was $70^{\circ}$ in 9 patients at 42 months follow-up, ROM was $85^{\circ}$ in 2 patients having single volar fragment. ${ }^{24}$ Authors of this series do caution about open technique in presence of comminution. The average range of motion achieved by the nail plate static traction device is comparable to results reported by other techniques.

Two of our patients were gentlemen of $>60$ years of age and their active range of motion of PIP joint at final follow-up at 25 and 30 months was $70^{\circ}$ and $65^{\circ}$, respectively. The relatively lower range of motion achieved in elderly patients in our series agrees with an observation made by Waris et al. ${ }^{21}$ that the range
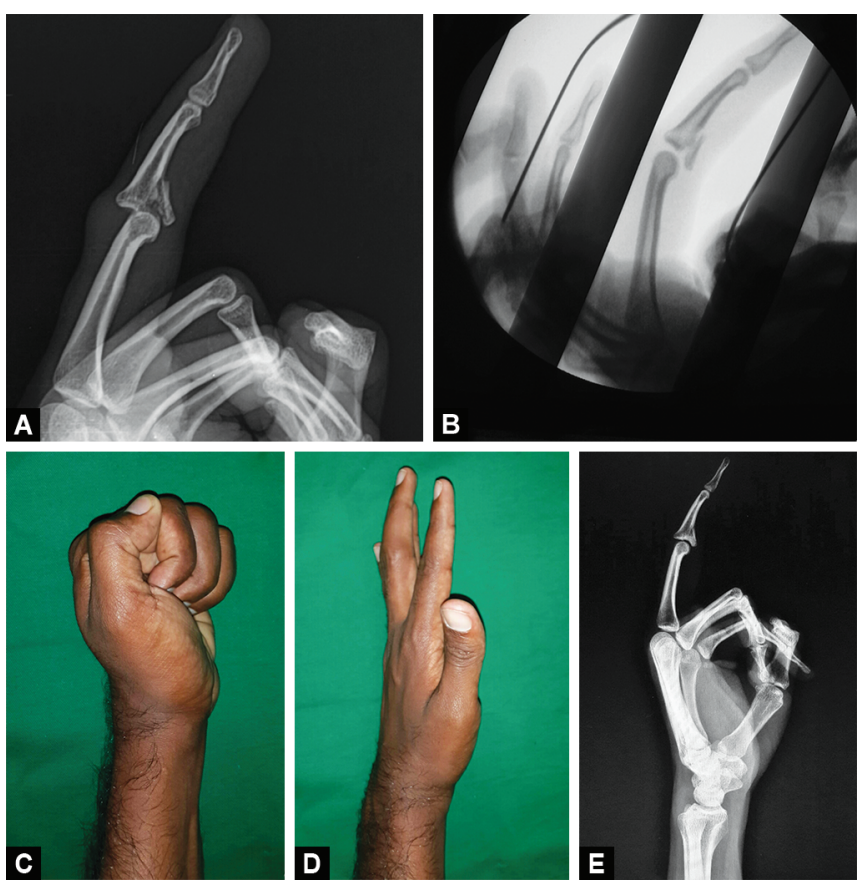

Figs 2A to E: (A) Lateral view of unstable fracture-dislocation of PIP joint at 1 day after injury. [Case 1 in Table 1] (B) Check view in image intensifier after application of splint, note alignment of joint surfaces and suboptimal position of the volar base fragment; (C and D) Range of motion at follow-up of 15 months post-injury. Note mild flexion deformity at PIP joint and complete extension at the DIP joint; (E) Postop radiograph at 15 months 
Static Traction and Early Mobilization Protocol for Intra-articular Fractures of PIP Joint

Table 2: Comparative data for similar injury from literature

\begin{tabular}{|c|c|c|c|c|}
\hline Study & Method of treatment & Number of patients & $\begin{array}{l}\text { Average duration } \\
\text { of follow-up }\end{array}$ & $\begin{array}{c}\text { Active flexion } \\
\text { achieved at PIP joint }\end{array}$ \\
\hline Morgan et al. ${ }^{6}$ & Dynamic digital traction & $\begin{array}{c}11 \text { (dorsal } \\
\text { dislocation + pilon fracture) }\end{array}$ & 24 months & $88^{\circ}$ \\
\hline Ellis et al. ${ }^{9}$ & $\begin{array}{l}\text { Dynamic external fixator } \\
\text { (pins and rubber bands) }\end{array}$ & 8 & 26 months & $88^{\circ}$ \\
\hline Ruland et al. ${ }^{10}$ & $\begin{array}{l}\text { Dynamic external fixator } \\
\text { (Pins and rubber bands) }\end{array}$ & 34 & 16 months & $93^{\circ}$ \\
\hline Waris et al. ${ }^{21}$ & Extension block pinning & 55 & 5.2 years & $80^{\circ}$ \\
\hline Badia et al. ${ }^{22}$ & Dynamic intradigital external fixator & 6 & 24 months & $89^{\circ}$ \\
\hline Newington et al. ${ }^{23}$ & Closed reduction $K$ wire fixation & 11 & 16 years & $85^{\circ}$ \\
\hline Hamilton et al. ${ }^{24}$ & Mini screw fixation & 9 & 42 months & $70^{\circ}$ \\
\hline Present study & $\begin{array}{l}\text { Static distraction device and early } \\
\text { mobilization }\end{array}$ & 11 & 17.3 months & $87.7^{\circ}$ \\
\hline
\end{tabular}

of motion achieved has a negative correlation with the age of the patient, although the number of patients in our series is too small to calculate statistical significance. Two of our patients presented late- at 10 days (Fig. 3 ) and 14 days after injury with lateral deviations at the fracture site. We followed our protocol for these delayed presentations as well. The range of motion achieved in them was $85^{\circ}$ (Fig. 3) and $45^{\circ}$ which was painless; there were persistent lateral deviations of $15^{\circ}$ and $20^{\circ}$, respectively, but there was no rotational deformity. The patient with pilon fracture had a lateral deviation of $20^{\circ}$ with a painless flexion arc of $65^{\circ}$ at the final follow-up (Fig. 4). After excluding these four cases (two patients $>60$ years and two late presentations) final mean range of motion achieved was $93.5^{\circ}$. Thus, the final range achieved was markedly better in acute cases in young patients. A similar finding was noted by De Smet and Boone in their study ${ }^{11}$ using Suzuki external fixator. Age of patient and time since injury seem to inversely correlate with the final range of motion achieved at the injured joint.

A residual flexion deformity or extension lag involving PIP and/or DIP joints is common after treatment of dorsal fracture- dislocation of PIP joints irrespective of treatment modality. ${ }^{5,10,21,24,25}$ In our series, two patients had flexion deformity and three patients had extension lag at the PIP joint. Five patients had extension lag at the DIP joint. Only 2 of the 11 cases had no extension lag or flexion deformity at the PIP or DIP joints. Their fracture pattern involved only 26 and $33 \%$ of the articular surface of the middle phalanx and could be considered stable as per the criteria described by Slade ${ }^{17}(<40 \%=$ stable; $>40 \%=$ unstable). So, it appears that the unstable fractures ( $>40 \%$ of articular surface involved) may leave a residual flexion deformity or lag at the PIP and/or DIP joint and patients must be counseled regarding this.

We observed that four out of five patients with DIP joint extension lag (mallet posture) had a complete extension of the PIP joint (Fig. 3) and the remaining one case had only $5^{\circ}$ of extension lag. Considering these findings, we believe that DIP extension lag is more likely to occur when the PIP joint recovers complete extension. We hypothesize that relative proximal migration of the dorsal base of the middle phalanx shortens central slip and induces slack in lateral bands. When the PIP joint recovers full extension this slack in lateral bands causes extension lag at the DIP joint. When there is a flexion deformity of the PIP joint, even if proximal migration of dorsal base of middle phalanx is present, length and tension in extensor apparatus are maintained, and DIP joint maintains complete extension (Fig. 2).
In unstable comminuted intra-articular fracture-dislocations of the PIP joint, anatomical reconstitution of the articular surface of the base of the middle phalanx, though ideal, is less important. ${ }^{26}$ Deitch et al. ${ }^{25}$ reported that despite significant radiographic abnormalities and decreased range of motion at PIP and DIP joints, patients had few functional deficits. Eighty-three percent of patients in their series reported little or no pain despite a high incidence $(96 \%)$ of posttraumatic degenerative changes. McAuliffe ${ }^{27}$ stated that residual articular step-off or gap, or central fragment depression is associated with the presence of degenerative changes on radiographs but it does not seem to adversely affect a range of motion, pain, or patient satisfaction, nor do results in these joints seem to deteriorate over time. In our series, anatomic restoration of the articular surface was possible in two cases with stable fractures having small volar base fragments. In the majority of cases, residual step in the articular surface was present, in six cases residual dorsal migration of axis of the middle phalan $x$ was also noted. Similar findings have been noted in other series as well. ${ }^{5,27}$ In such cases, the middle phalanx heals with an expanded base in the dorsal palmar direction with deepening, creating a concavity in the base of the phalanx to allow gliding motion at the joint, ${ }^{11}$ as noted in Figure 4 of our case. Despite these findings, only three patients gave a history of mild pain on terminal flexion especially when passive flexion beyond available active range was attempted. All of them had no trouble in day-to-day activities and declined prescription of analgesics.

There are several limitations of this study. The number of cases in our study was small and there was a lack of a comparison group. Loss of $40 \%(8 / 20)$ of patients to follow-up is another limitation of the study. Although physicians know these injuries as challenging, patients often regard finger injuries as minor problems, and especially after regaining painless, reasonable range of motion of the injured finger, it is difficult to get patients back for evaluation of long-term results.

The disadvantages of this technique include the need for good patient compliance for assembly care in the first 2 weeks and a learning curve for correct application of the traction system. Possible complications include loss of nail plate due to excessive traction. In our series, we noted this in one patient at 2 weeks when he reported for removal of the traction. However, there was no long-term deformity, and the appearance of the finger was normal after nail plate regeneration. Lateral deviation of the 

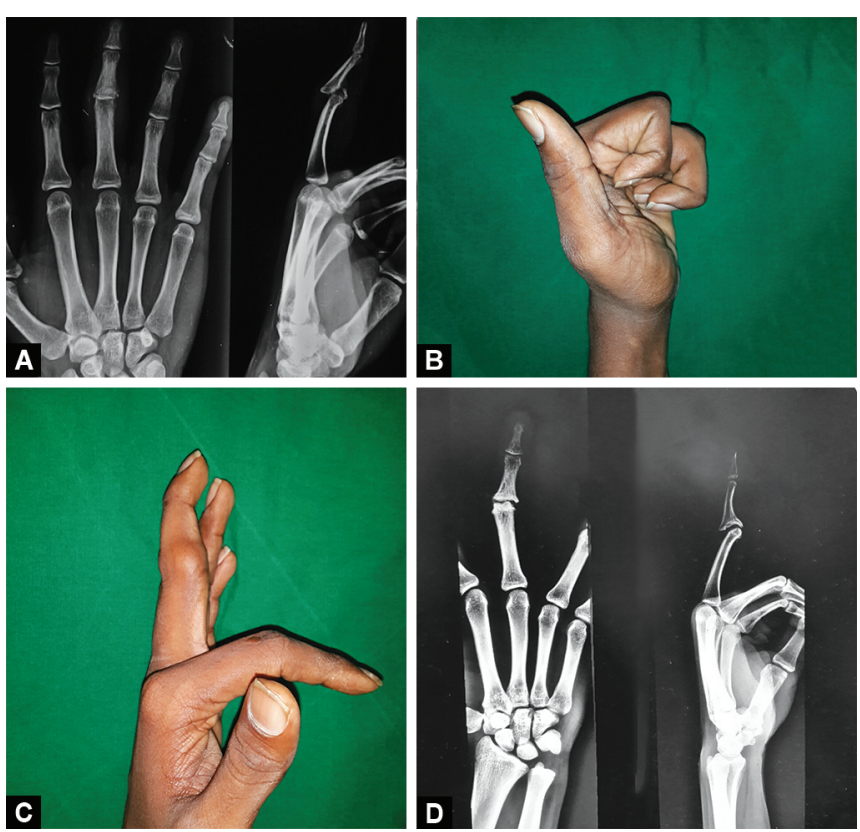

Figs 3A to D: (A) Preoperative radiograph of a 10-day-old injury in a 21-year-old patient. [Case 10 in Table 1] (B and C) Range of motion at 31 months post-injury, note mild hyperextension at PIP joint and extension lag at the DIP joint; (D) Radiograph at 31 months post-injury. Lateral deviation noted in the coronal film, in lateral proximal migration of dorsal base, and remodeling of middle phalanx base can be appreciated. Despite suboptimal radiograph, patient had no pain

finger at the fracture site is possible if the splint is not oriented in the same sagittal plane as the injured finger.

The presence of a nail bed injury precludes utilization of this method and associated forearm or wrist injuries need to be stabilized first before application of traction assembly. Associated fractures in other bones in hand are a relative contraindication for this technique.

\section{Conclusion}

We believe that the static traction technique with early mobilization is a safe, relatively simple, and effective technique in the management of these difficult injuries. Early mobilization of joint seems to facilitate remodeling of the articular base of the middle phalanx with the recovery of painless motion at the PIP joint despite radiological abnormalities.

\section{References}

1. Glickel SZ, Barron OA. Proximal interphalangeal joint fracture dislocations. Hand Clin 2000;16(3):333-344. DOI: 10.1016/S0749-0712(21)00575-8

2. Venkataswami R. Surgery of the injured hand-Towards functional restoration. 1st ed., ch. 32. Jaypee Brothers Medical Publishers (P) Ltd.; 2009. pp. 405-408.

3. Mc Elfresh BC, Dobyns JH, O' Brien BT. Management of fracturedislocations of the proximal interphalangeal joints by extension block splinting. J Bone Joint Surg 1972;54(8):1705-1711. DOI: 10.2106/00004623-197254080-00010

4. Viegas SF. Extension block pinning for proximal interphalangeal joint fracture-dislocations: a preliminary report of a new technique. J Hand Surg 1992;17(5):896-901. DOI: 10.1016/0363-5023(92)90464-z

5. Schenck RR. Dynamic traction and early passive movement for fractures of the proximal interphalangeal joint. J Hand Surg Am 1986;11(6):850-858. DOI: 10.1016/s0363-5023(86)80236-2
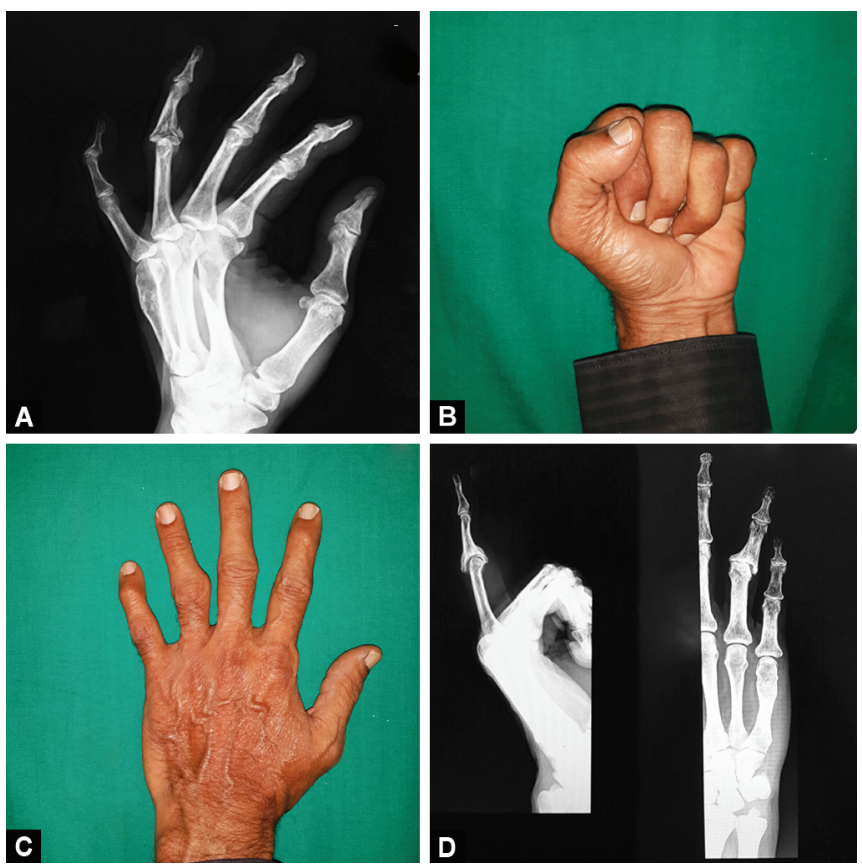

Figs 4A to D: (A) Pilon fracture of middle phalanx base in a 61-year-old man. [Case 9 in Table 1] (B and C) Final range of motion at 30 months post-injury; (D) Radiographs at 30 months post-injury demonstrate lateral deviation of injured finger and significant remodeling of the base of the middle phalanx. The patient reported no pain in the involved finger and was satisfied with the result

6. Morgan JP, Gordon DA, Klug MS, et al. Dynamic digital traction for unstable comminuted intra-articular fracture-dislocations of the proximal interphalangeal joint. J Hand Surg Am 1995;20(4):565-573. DOI: 10.1016/S0363-5023(05)80268-0

7. Agee JM. Unstable fracture- dislocations of the proximal interphalangeal joint of the fingers: a preliminary report of a new treatment technique. J Hand Surg Am 1978;3(4):386-389. DOI: 10.1016/s0363-5023(78)80045-8

8. Slade JF, Baxamusa TH, Wolfe SW. External fixation of proximal interphalangeal joint fracture-dislocations. Atlas Hand Clin 2000;5:1- 29.

9. Ellis SJ, Cheng R, Prokopis $P$, et al. Treatment of proximal interphalangeal dorsal fracture-dislocation injuries with dynamic external fixation: a pins and rubber band system. J Hand Surg Am 2007;32(8):1242-1250. DOI: 10.1016/j.jhsa.2007.07.001

10. Ruland RT, Hogan CJ, Cannon DL, et al. Use of dynamic distraction external fixation for unstable fracture-dislocations of the proximal interphalangeal joint. J Hand Surg Am 2008;33(1):19-25. DOI: 10.1016/j.jhsa.2007.07.018

11. De Smet L, Boone P. Treatment of fracture - dislocation of the proximal interphalangeal joint using the Suzuki external fixator. J Orthop Trauma 2002;16(9):668-671. DOI: 10.1097/00005131200210000-00009

12. Deshmukh SC, Kumar D, Mathur K, et al. Complex fracture-dislocation of the proximal interphalangeal joint of the hand. Results of a modified pins and rubbers traction system. J Bone Joint Surg Br 2004;86(3):406-412. DOI: 10.1302/0301-620x.86b3.14350

13. Eaton RG, Malerich MM. Volar plate arthroplasty of the proximal interphalangeal joint: a review of ten years' experience. J Hand Surg 1980;5(3):260-280. DOI: 10.1016/s0363-5023(80)80011-6

14. Wolfe SW, Katz LD. Intra-articular impaction fractures of the phalanges. J Hand Surg Am 1995;20(2):327-333. DOI: 10.1016/S0363-5023(05)80035-8

15. Stern PJ, Roman RJ, Kiefhaber TR, et al. Pilon fractures of the proximal interphalangeal joint. J Hand Surg Am 1991;6(5):844-850. DOI: 10.1016/s0363-5023(10)80147-9 
16. Williams RMM, Kiefhaber TR, Sommerkamp TG, et al. Treatment of unstable dorsal proximal interphalangeal joint fracture/dislocations using a hemi-hamate autograft. J Hand Surg Am 2003;28(5):856-865. DOI: 10.1016/s0363-5023(03)00304-6

17. Merrell G, Slade JF. Green's operative hand surgery. 6th ed., ch. 9, Livingstone, PA: Elsevier Churchill; 2011. p. 294.

18. Calfee RP, Sommerkamp TG. Fracture dislocations about the finger joints. J Hand Surg Am 2009;34(6):1140-1147. DOI: 10.1016/j. jhsa.2009.04.023

19. Hamada $Y$, Hibino N, Tonogai I, et al. Staged external fixation for chronic fracture-dislocation of proximal interphalangeal joint: outcomes of patients with minimum 2-year follow up. J Hand Surg Am 2012;37(3):434-439. DOI: 10.1016/j.jhsa.2011.11.005

20. Stark RH. Treatment of difficult PIP joint fractures with a mini-external fixation device. Ortho Rev 1993;22(5):609-615.

21. Waris E, Mattila S, Sillat T, et al. Extension block pinning for unstable proximal interphalangeal joint dorsal fracture dislocations. J Hand Surg Am 2016;41(2):196-202. DOI: 10.1016/j.jhsa.2015.11.007

22. Badia A, Riano F, Ravikoff J, et al. Dynamic intradigital external fixation for proximal interphalangeal joint fracture dislocations. J Hand Surg Am 2005;30(1):154-160. DOI: 10.1016/j.jhsa.2004.07.019
23. Newington DP, Davis TR, Barton NJ. The treatment of dorsal fracture-dislocation of the proximal interphalangeal joint by closed reduction and Kirschner wire fixation: a 16-year follow up. J Hand Surg Br 2001;26(6):537-540. DOI: 10.1054/jhsb.2001.0698

24. Hamilton SC, Stern PJ, Fassler PR, et al. Mini-screw fixation for the treatment of proximal interphalangeal joint dorsal fracturedislocations. J Hand Surg Am 2006;31(8):1349-1354. DOI: 10.1016/j. jhsa.2006.07.011

25. Deitch MA, Kiefhaber TR, Comisar BR, et al. Dorsal fracture dislocations of the proximal interphalangeal joint: surgical complications and long-term results. J Hand Surg Am 1999;24(5):914-923. DOI: 10.1053/jhsu.1999.0914

26. Kang R, Stern PJ. Fracture dislocations of the proximal interphalangeal joint. J Hand Surg Am 2002;2(2):47-59. DOI: 10.1053/jssh. 2002.33317

27. McAuliffe JA. Dorsal fracture dislocation of the proximal interphalangeal joint. J Hand Surg Am 2008;33(10):1885-1888. DOI: 10.1016/j.jhsa.2008.08.017 